\title{
Scrotal granulomatous aspergillosis in a dromedary camel (Camelus dromedarius)
}

\author{
Frine Eleonora Scaglione ${ }^{1 *}$, Andrea Peano ${ }^{1}$, Sara Piga² ${ }^{2}$ Stefano Meda ${ }^{2}$ Enrico Bollo ${ }^{1}$, Francesca Tiziana Cannizzo ${ }^{1}$, \\ Mario Pasquetti ${ }^{1}$ and Henrik Elvang Jensen ${ }^{3}$
}

\begin{abstract}
Background: This report describes a case of primary subcutaneous aspergillosis in a 7-year-old neutered male dromedary camel (Camelus dromedarius).

Case presentation: The animal developed a large nodular lesion in the right scrotum two years after surgical intervention for neutering. The mass had a firm consistency and was painful at palpation. Histopathology revealed dermal granulomatous inflammation with a necrotic centre, surrounded by plasma cells, macrophages, neutrophils, and sparse fungal hyphae characterised by parallel cell walls, distinct septa, and dichotomous branching. Fungal culture was not performed, but a panel of mono- and polyclonal antibodies specific for different fungal genera identified the hyphae as Aspergillus sp.
\end{abstract}

Conclusions: The occurrence of subcutaneous lesions is a rare manifestation of aspergillosis in animals, and this appears to be the first case reported in the dromedary camel.

Keywords: Dromedary, Camelids, Immunohistochemistry, Aspergillus, Scrotum

\section{Background}

Aspergillus species are ubiquitous environmental fungal organisms [1]. They have been found worldwide in humans, in almost all domestic animals, birds as well as in many wildlife species, causing a wide range of diseases from localized infections to fatal disseminated forms [2]. Invasive aspergillosis in humans is typically associated with pulmonary infection in immunosuppressed patients [3]. However, a number of extrapulmonary localizations has been reported, sometimes in immunocompetent individuals [1]. Cutaneous aspergillosis occurs relatively less frequently, as either primary or secondary infection $[3,4]$. Primary cutaneous aspergillosis usually involves sites of skin injury of various nature, while secondary lesions result either from contiguous extension to the skin from infected underlying structures or from widespread blood-borne infections [3].

In the same way aspergillosis in animals is mainly a disease of the respiratory tract (nasal cavities and lungs in mammals; trachea, lungs and air sacs in birds), although

\footnotetext{
* Correspondence: frineeleonora.scaglione@unito.it

${ }^{1}$ Dipartimento di Scienze Veterinarie, Università degli Studi di Torino, Largo

Paolo Braccini 2, 10095 Grugliasco (To), Italy

Full list of author information is available at the end of the article
}

other localizations typical of particular hosts have been recognized (e.g. the guttural pouches and the cornea in horses; the retro-orbital space in cats; the intervertebral disk in dogs) [2]. Cases with cutaneous involvement are hardly found in the literature [2]. The present report describes a case of primary subcutaneous aspergillosis in a dromedary camel (Camelus dromedarius).

\section{Case presentation}

A captive 7-year-old neutered male dromedary camel, living in a zoological garden in northern Italy (Zoom Torino) and sharing the habitat with two conspecifics (a male and a female), developed a subcutaneous nodular mass in the right scrotum. The animal had been neutered two years before. A blood sample was taken from the left jugular vein and a full blood screening was performed. Differential diagnoses taken in account included an abscess and a neoplastic process. The animal was treated with antibiotics (Enrofloxacin2,5 $\mathrm{mg} / \mathrm{kg}$ ) given orally for 2 weeks and with anti-inflammatory drugs (Meloxicam 0,4 mg/kggiven orally for 7 days. After two months, the mass had increased (about $10 \mathrm{~cm}$ in diameter) (Fig. 1). At that time, it was decided to proceed with surgery. The animal was anaesthetized 


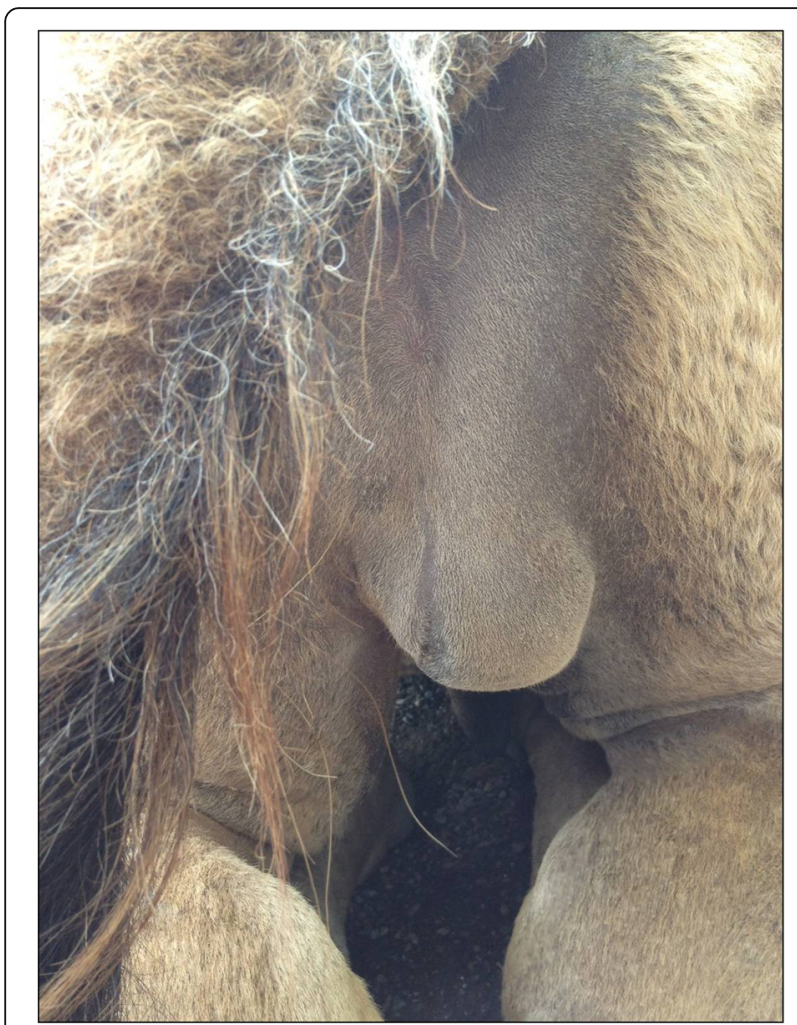

Fig. 1 Clinico-pathological evidence of the mass in the scrotum of a dromedary camel (Camelus dromedarius). Subcutaneous nodular mass in the scrotum of a dromedary camel (Camelus dromedarius)

with a xylazine-ketamine combination and the surgical area was scrubbed with chlorhexidine solution. Both the mass and the capsule were removed. The scrotum was flushed with sterile saline solution and a drainage was placed to prevent fluid accumulation. The drainage was removed after four days, and after seven days the antibiotic therapy was discontinued. The mass (Fig. 2) was fixed in $10 \%$ neutral buffered formalin and referred to the Department of Veterinary Sciences of the University of Turin (Italy) for histological and histochemical examination. The mass was paraffin-embedded and sections of $4 \mu \mathrm{m}$ were stained with hematoxylin and eosin.

Because fungal cultures were not performed at the time of surgery, an immunohistochemical study was carried out in order to identify of the organism. Sections of the mass were mounted on adhesive slides and kept at $+4{ }^{\circ} \mathrm{C}$ until processed. Sections were examined with a panel of mono- and polyspecific anti-fungal reagents applied in various immunohistochemical techniques. In the techniques, primary reagents for immunostaining included genus-specific monoclonal and polyclonal antibodies reacting with species of Aspergillus, Mucorales, Candida, Geotrichum, Fusarium and Scedosporium, respectively [5-7]. The detecting system PowerVision + (Part

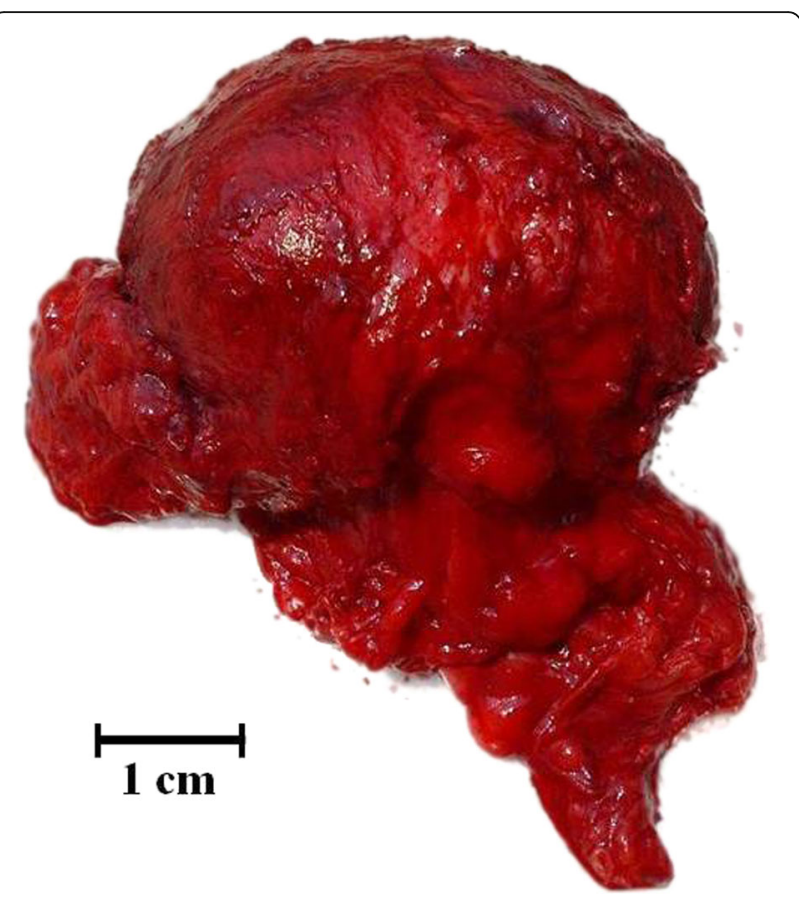

Fig. 2 Macroscopical appearance of the mass. Encapsulated mass removed out of the scrotum of a dromedary camel

No. DPVB +110AEC; Immuno Vision Technologies Co. USA) was used for signal-amplification and applied according to the manufacturer's instructions. Sections were counterstained in Meyer's hematoxylin for $10 \mathrm{~s}$, and washed for $1 \mathrm{~min}$ in tap water and $4 \mathrm{~min}$ in distilled water. Finally, sections were mounted with glycerol-gelatin. To ensure specific reactivity of antibodies, sections were run in parallel with sections from laboratory animals experimentally infected with homologous and heterologous fungi. Moreover, in all series of staining, negative controls were run without primary reagents and with substitution of the primary antiserum or monoclonal antibody of identical isotypes, respectively, raised towards nonsense antigens.

\section{Results}

At palpation the mass had a firm consistency and was painful. It measured $4 \mathrm{~cm}$ in diameter and was firmly adherent to the inner surface of the scrotum. Haematology indicated leukocytosis suggestive of a chronic inflammation, while no biochemical abnormality was detected. At the opening of the scrotum through a vertical incision, the mass appeared to be surrounded by a capsule and showed a solid appearance at the cut surface. Histopathology revealed dermal granulomatous inflammation with a necrotic centre, surrounded by plasma cells, macrophages, neutrophils, and sparse fungal hyphae. The mass was surrounded by a thick fibrous 
layer with multifocal lymphocytic infiltrate and haemorrhages. In order to improve the visualization of the fungal elements, periodic acid-Schiff and Grocott stains were applied to selected sections of the mass. Hyphae were characterised by parallel cell walls, distinct septa, and a dichotomous branching pattern (Figs. 3 and 4). Fungal hyphae were present only in the encapsulated granulomatous mass and no invasion of the epidermis and adjacent structures has been observed. Within the nodule removed from the dromedary the fungal hyphae only stained positive (Fig. 5) with the monoclonal antibody (clone WF-AF-1, LSBio) reacting specifically with Aspergillus [7, 8]. At follow up, one year after the excision of the mass, the animal was in good health with no sign of recurrence of infection.

\section{Discussion}

This case of aspergillosis appears to be the first with a subcutaneous localization ever reported in the dromedary camel, and represents a rarity with regards to aspergillosis in animals [2]. In the dromedary, Aspergillus has been found associated to post-traumatic buccal infections and osteomyelitis in consequence of mandibular fractures [9] and to a clinical syndrome with multiorganic involvement, skin excluded [10]. Cases of pulmonary aspergillosis have also been reported in other camelids, such as alpaca [11], and lama [12].

Given the localization of the mass, the present case had likely a traumatic origin, represented by the surgical procedure employed to neuter the animal. The subcutaneous tissues exposed during surgery were probably colonized by spores of Aspergillus that induced a slow granulomatous reaction. The granulomatous inflammation with abundant fibrosis is a typical finding of chronic

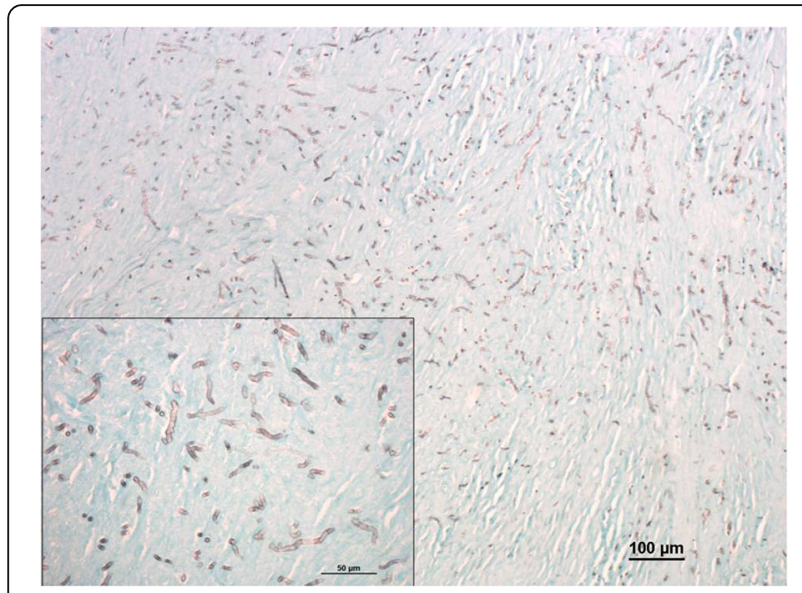

Fig. 3 Histopathology of the mass (Grocott). Histopathology of a scrotal mass removed from a dromedary camel. Fungal hyphae characterized by parallel cell walls, distinct septa, and dichotomous branching (Grocott staining)

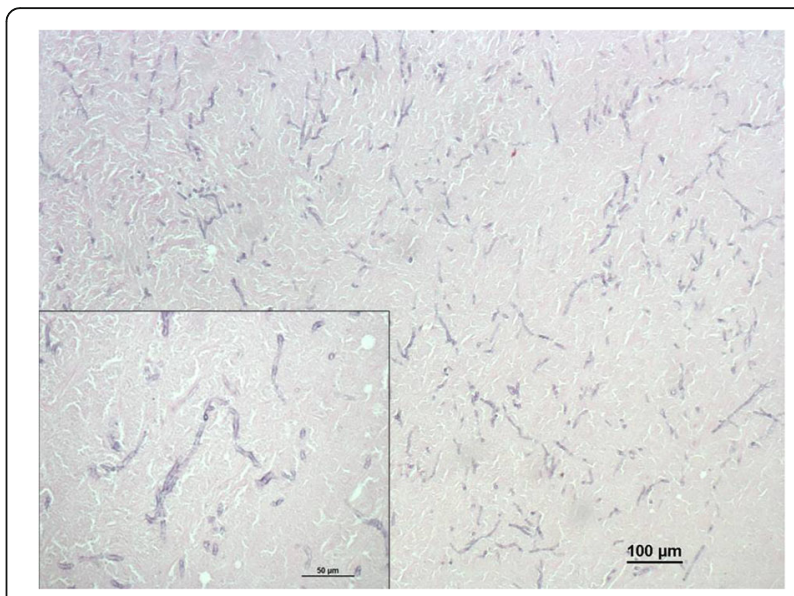

Fig. 4 Histopathology of the mass (HE). Histopathology of a scrotal mass removed from a dromedary camel. Fungal hyphae characterized by parallel cell walls, distinct septa, and dichotomous branching (HE staining)

aspergillosis [13]. Reports in human medicine frequently describe a more rapid development of different lesions, represented by erythematous to violaceous papules or plaques that may progress to form necrotic ulcers with a central eschar, pustules and subcutaneous abscesses $[14,15]$. However, also infections characterized by subcutaneous chronically slow-growing nodules have been reported [3]. In the present case it is somewhat surprising that two years after surgery the nodular mass grew to only $4 \mathrm{~cm}$ diameter and then a further $6 \mathrm{~cm}$ diameter in just two months. However, cases with a similar trend have been already described in human medicine. For example Cheetham [16] reported a patient with a subcutaneous infection by Aspergillus attributed to the inoculation of fungal spores occurred

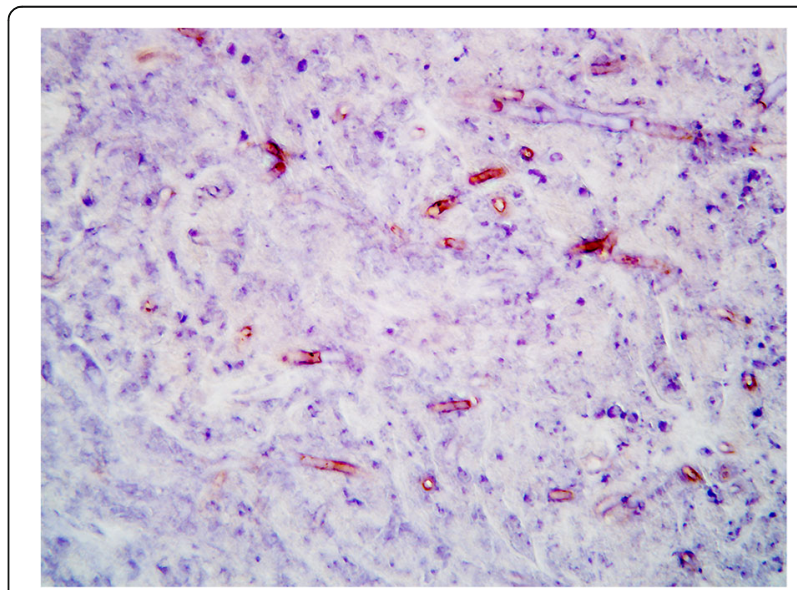

Fig. 5 Immunohistochemical features of the mass. Specific immunolabelling of Aspergillus sp. in the lesion from a dromedary camel 
about one and a half year before during a course of penicillin injections. The authors speculated that the fungus lay dormant, or only developed slowly, during the year before lesion was first noted, and that the more rapid development of the mass in the later stages may have been due to the appearance of a mutant more adapted to growth in the body than the original strain [16]. Even more extreme is the case described by Lakhanpal et al. [14], regarding a 30-year-old male who developed a subcutaneous nodule due to Aspergillus infection over a period of 15 years.

As noted above, cases of aspergillosis with cutaneous involvement are rarely found in veterinary literature. In cats, the occurrence of skin nodules has been reported as the result of contiguous extension to the skin from infected underlying structures (nasal cavity and retroorbital space) [2, 17]. In other cases, mostly regarding birds, the suspected pathogenesis was more similar to that of our case, i.e. on traumatic base. For example, Abrams et al. [18] described a case of Aspergillus blepharitis and dermatitis in a Peregrine Falcon-Gyrfalcon Hybrid (Falco peregrinus x Falco rusticolus) likely due to a trauma occurred during hunting. Copetti et al. [13] reported an outbreak of aspergillosis in some Pekin mallards (Anas platyrhynchos), with the involvement of different organs including the skin. The skin presented multiple elevated, yellowish brown, crusted, multifocal lesions located at the base of the feather follicles in the breast. The infection was attributed to skin trauma incited by the poultry litter [13]. Chute et al. [19] described a systemic fatal infection by Aspergillus fumigatus in White Rock cockerels, that was attributed to the contamination of the cutaneous incision performed to caponize the animals. Finally, in a case of some chickens, cutaneous aspergillosis was seen with generalized, tumor-like swellings, the infection being limited to the skin [20].

Primary "traumatic" aspergillosis is a well characterized condition, although rare if compared with pulmonary and systemic infections, in human medicine $[3,4]$. Lesions usually develop in cutaneous sites traumatized in course of accidents or burns, but also due to surgery or minor medical procedures (positioning of intravenous catheter, drug injections, use of occlusive dressings and tapes, etc.) [3]. Most of the cases occur in patients with deficiencies in their immunological status of various reasons (AIDS, corticosteroids use, chemotherapy and immunosuppressive therapy used in organ transplants, diabetes, neonatal period and hematological disorders) [21], although primary cutaneous aspergillosis has been reported also in immunocompetent individuals [15].

\section{Conclusions}

Although culture isolation remains the gold standard for the identification of fungi involved in human and animal infections [11], many cases are not cultured because a fungal etiology is not suspected when biological samples are sent to the laboratory [5, 6]. Even when submitted, cultures often fail to isolate the fungus responsible of the infection [8]. Despite the lack of culture in the present case, a definitive diagnosis of aspergillosis was upheld by the application of specific immunohistochemical staining techniques.

\section{Acknowledgements \\ The authors gratefully acknowledge the Centro di Referenza di Patologia Comparata "Bruno Maria Zaini". The case was presented as poster at the congress of the European Association of Zoo and Wildlife Veterinarians, Vienna (Austria), 8-11 May 2013.}

\section{Funding}

No funding source has been employed to perform this research.

\section{Availability of data and material}

All the data are presented in the paper and there are no additional supporting files.

\section{Authors' contributions}

FES was the mentor and principal advisor and proposed the concept of the study, EB and FTC performed histological examination and diagnosis, HEJ performed immunohistochemical investigation, SP and SM were responsible for the surgical procedure. AP and MP were involved in the drafting and writing of the manuscript. All authors have read, commented and approved the final article.

\section{Authors' information}

Not applicable.

\section{Competing interests}

None of the authors of this paper has a financial or personal relationship with other people or organisations that could inappropriately influence or bias the content of the paper.

\section{Consent for publication}

Not applicable.

Ethics approval and consent to participate

The surgical procedure was performed after the agreement of the owner.

\section{Author details}

'Dipartimento di Scienze Veterinarie, Università degli Studi di Torino, Largo Paolo Braccini 2, 10095 Grugliasco (To), Italy. ${ }^{2}$ Zoom Torino, Strada Piscina 36, 10040 Cumiana, Italy. ${ }^{3}$ Department of Veterinary Disease Biology, University of Copenhagen, Ridebanevej 3, DK-1870 Frederiksberg C, Copenhagen, Denmark.

Received: 9 March 2016 Accepted: 25 March 2017

Published online: 29 March 2017

\section{References}

1. Paterson DL. New clinical presentations of invasive aspergillosis in nonconventional hosts. Clin Microbiol Infect. 2004;10:24-30.

2. Seyedmousavi S, Guillot J, Arné P, de Hoog GS, Mouton JW, Melchers WJG, Verweij PE. Aspergillus and aspergilloses in wild and domestic animals: a global health concern with parallels to human disease. Medical Mycol. 2015;53:765-97.

3. Van Burik JA, Colven R, Spach DH. Cutaneous Aspergillosis. J Clin Microbiol. 1998;36:3115-21.

4. Perusquía-Ortiz AM, Vázquez-González D, Bonifaz A. Opportunistic filamentous mycoses: aspergillosis, mucormycosis, phaeohyphomycosis and hyalohyphomycosis. J Dtsch Dermatol Ges. 2012;10:611-21.

5. Jensen H, Schonheyder HC, Hotchi M, Kaufman L. Diagnosis of systemic mycosis by specific immunohistochemical tests. APMIS. 1996;104:241-58. 
6. Jensen HE, Salonen J, Ekfors TO. The use of immunohistochemistry to improve sensitivity and specificity in the diagnosis of systemic mycosis in patients with haematological malignancies. J Pathol. 1997;181:100-5.

7. Jung J, Park YS, Sung H, Song JS, Lee S-O, Choi S-H, Kim YS, Woo JH, Kim S-H Using immunohistochemistry to assess the accuracy of histomorphologic diagnosis of aspergillosis and mucormycosis. Clin Infect Dis. 2015;11:1664-70

8. Choi JK, Mauger J, McGowan L. Immunohistochemical detection of Aspergillus species in pediatric tissue samples. Am J Clin Pathol. 2004;121:18-25.

9. Al-Dughaym AM, Ramadan RO, Mohamed GE, Fadlelmula A, Abdin-Bey MR. Post-traumatic buccal infection and osteomyelitis associated with mandibular fractures in the dromedary camel. J camel pract res. 2003;10:57-60.

10. El-Khouly AB, Gadir FA, Cluer DD, Manefield GW. Aspergillosis in camels affected with a specific respiratory and enteric syndrome. Aust Vet J. 1992;69:182-6.

11. Hughes $\mathrm{K}$, Mueller K. Pathologic lesions of mycotic pneumonia in an alpaca following third compartment ulceration. J Vet Diagn Investig. 2008;20:672-5.

12. Quist CF, Dutton DM, Schneider DA, Prestwood AK. Gastrointestinal ulceration and pulmonary aspergillosis in a llama treated for parelaphostrongylosis. J Am Vet Med Assoc. 1998;212:1438-41.

13. Copetti MV, Barcelos Ada S, Kommers GD, Santurio JM, Oliveira FN, Lovato M. Cutaneous, respiratory and hepatic aspergillosis in Brazilian white Pekin mallards (Anas platyrhynchos). Mycopathologia. 2015;179:321-5.

14. Lakhanpal S, Pandhi RK, Khaitan BK, lyer VK, Bannerjee U. Primary cutaneous aspergillosis in an immunocompetent host. Acta Derm Venereol. 2000;80:74-5.

15. Venugopal TV, Venugopal PV. Primary cutaneous aspergillosis from Tamilnadu diagnosed by fine needle aspiration cytology. Med Mycol Case Rep. 2012;1:103-6.

16. Cheetham HD. Subcutaneous infection due to Aspergillus terreus. J Clin Pathol. 1964;17:251-3.

17. Giordano C, Gianella P, Bo S, Vercelli A, Giudice C, Della Santa D, Tortorano AM, Peruccio C, Peano A. Invasive mould infections of the naso-orbital region of cats: a case involving Aspergillus fumigatus and an aetiological review. J Feline Med Surg. 2010;12:714-23.

18. Abrams GA, Paul-Murphy J, Ramer JC, Murphy CJ. Aspergillus Blepharitis and Dermatitis in a Peregrine Falcon-Gyrfalcon Hybrid (Falco peregrines $x$ Falco rusticolus). J Avian Med Surg. 2001;15(2):114-20.

19. Chute HL, Witter JF, Rountree JL. O' meara DC. The pathology of a fungous infection associated with a caponizing injury J Am Vet Med Assoc. 1955;127:207-9.

20. Mohammed MA, Sokkar SM, Batrawi A, Refaie MK, Ahmed AA. Natural and experimental nodular dermatomycosis in chickens. Avian Pathol. 1980;9:185-92.

21. Galimberti R, Kowalczuk A, Hidalgo Parra I, Gonzalez Ramos M, Flores V. Cutaneous aspergillosis: a report of six cases. Br J Dermatol. 1998;139:522-6.

\section{Submit your next manuscript to BioMed Central and we will help you at every step:}

- We accept pre-submission inquiries

- Our selector tool helps you to find the most relevant journal

- We provide round the clock customer support

- Convenient online submission

- Thorough peer review

- Inclusion in PubMed and all major indexing services

- Maximum visibility for your research

Submit your manuscript at www.biomedcentral.com/submit
Biomed Central 